\title{
Intentional right atrial exit for microcatheter infusion of pericardial carbon dioxide or iodinated contrast to facilitate sub- xiphoid access
}

\author{
Toby Rogers, BM, BCh, MRCP*, Kanishka Ratnayaka, MD ${ }^{\star}$, , William H. Schenke, BA*, \\ Anthony Z. Faranesh, PhD $^{\star}$, Jonathan R. Mazal, MS ${ }^{\star}$, William W. O’Neill, MD ${ }^{\ddagger}$, Adam B. \\ Greenbaum, MD $\ddagger$, and Robert J. Lederman, MD $^{*}$ \\ "Cardiovascular and Pulmonary Branch, Division of Intramural Research, National Heart Lung \\ and Blood Institute, National Institutes of Health, Bethesda, MD, USA \\ †Department of Cardiology, Children's National Medical Center, Washington, DC, USA \\ \#Institute for Structural Heart Disease, Division of Cardiology, Henry Ford Health System, Detroit, \\ MI, USA
}

\begin{abstract}
Objectives-We test the safety of trans-atrial pericardial access using small catheters, infusion of carbon dioxide $\left(\mathrm{CO}_{2}\right)$ or iodinated contrast to facilitate sub-xiphoid access, and catheter withdrawal under full anticoagulation.

Background-Sub-xiphoid pericardial access is required for electrophysiological and structural heart interventions. If present, an effusion protects the heart from needle injury by separating the myocardium from the pericardium. However, if the pericardium is 'dry' then there is a significant risk of right ventricle or coronary artery laceration caused by the heart beating against the needle tip. Intentional right atrial exit is an alternative pericardial access route, through which contrast media could be infused to separate pericardial layers.
\end{abstract}

Methods-Trans-atrial pericardial access was obtained in a total of 30 Yorkshire swine using 4Fr or $2.8 \mathrm{Fr}$ catheters. In 16 animals, trans-atrial catheters were withdrawn under anticoagulation and MRI was performed to monitor for pericardial hemorrhage. In 14 animals, iodinated contrast or $\mathrm{CO}_{2}$ was infused before sub-xiphoid access was obtained.

Results-Small effusions (mean $18.5 \mathrm{~mL}$ ) were observed after 4Fr (1.3mm outer-diameter) but not after $2.8 \mathrm{Fr}(0.9 \mathrm{~mm}$ outer-diameter) trans-atrial catheter withdrawal despite full anticoagulation (mean activated clotting time $383 \mathrm{sec}$ ), with no hemodynamic compromise. Pericardial $\mathrm{CO}_{2}$ resorbed spontaneously within $15 \mathrm{~min}$.

Conclusions-Intentional trans-atrial exit into the pericardium using small catheters is safe and permits infusion of $\mathrm{CO}_{2}$ or iodinated contrast to separate pericardial layers and facilitate subxiphoid access. This reduces the risk of right ventricular or coronary artery laceration. $2.8 \mathrm{Fr}$ trans-

Address for correspondence: Robert J. Lederman, MD, National Heart Lung and Blood Institute, National Institutes of Health, Building 10, Room 2c713, Bethesda, MD 20892-1538, USA. Telephone: +1-301-402-6769. lederman@ nih.gov. 
atrial catheter withdrawal does not cause any pericardial hemorrhage, even under full anticoagulation.

\section{Keywords}

Pericardial access; Epicardial intervention; Trans-atrial puncture; Structural heart intervention; Electrophysiology

\section{Introduction}

Access to the naïve or 'dry' pericardial space in the absence of effusion is required for interventional procedures, for example epicardial mapping and radiofrequency ablation for ventricular tachycardia and for left atrial appendage suture ligation [1-3]. Sub-xiphoid access is usually obtained under fluoroscopic guidance. A needle is advanced slowly towards the heart and aliquots of iodinated contrast are injected until the tip is confirmed inside the pericardial space. A soft-tipped wire is then introduced through the needle, over which larger sheaths can be exchanged. Even though 18-gauge (1.3mm outer diameter) needle entry into the right ventricle is considered benign [4], there is a risk of laceration to the right ventricle or epicardial coronary arteries. Torsional cardiac and translational respiratory motion exacerbate the risk of more serious injury by laceration as the heart beats against the sharp needle tip. Bleeding complication rates of 4-7\% have been reported in single and multicenter studies [5-7] but real world complication rates may be higher [8-10].

An alternative trans-atrial approach for pericardial access, by intentionally puncturing through the right atrial appendage (RAA), was first proposed for diagnostic sampling and delivery of drugs [11]. A catheter is navigated to the tip of the RAA from the inferior vena cava. Next a needle or guidewire is used to puncture through the RAA wall into the pericardial space. Animal experiments have demonstrated that pericardial effusions did not develop after needle withdrawal and the puncture site healed rapidly [12]. Importantly, this approach appeared technically easy and safe with no evidence of injury to epicardial vessels or to the thin-walled right ventricle. Other groups have proposed this approach for epicardial pacing wire implantation [13] or epicardial mapping and ablation [14]. We have advocated trans-atrial access to the pericardium to achieve other therapeutic procedures, such as intrapericardial tricuspid annuloplasty [15].

We hypothesized that despite aggressive anticoagulation, atrial tissue recoil would avoid significant pericardial hemorrhage or effusion after trans-atrial access used to introduce a small catheter into the 'dry' pericardium. Second, contrast media could then be infused through this catheter to separate visceral and parietal pericardial layers and enhance the safety of sub-xiphoid access with larger sheaths for electrophysiological or heart interventions. Third, we compared pericardial infusion of carbon dioxide versus iodinated contrast, reasoning that gas would separate the pericardial walls preferentially at the anterior site of supine sub-xiphoid access, and further that gas might spontaneously resorb as a margin of safety. 


\section{Methods}

Procedures were approved by the institutional animal care and use committee. Yorkshire swine were anesthetized with ketamine $(25 \mathrm{mg} / \mathrm{kg})$, midazolam $(15 \mathrm{mg} / \mathrm{kg})$ and glycopyrrolate $(0.01 \mathrm{mg} / \mathrm{kg})$, and maintained on sevoflurane (1-4\%) with mechanical ventilation, and femoral arteries and veins were accessed. Experiments took advantage of a combined interventional cardiovascular magnetic resonance imaging (MRI) and biplane Xray fluoroscopy suite (1.5T Aera and Artis Zee, Siemens, Erlangen, Germany) for characterization of pericardial effusion. Pericardial access via RAA puncture was obtained in a total of 30 Yorkshire swine with mean weight $48 \mathrm{~kg}$ (range $27-66 \mathrm{~kg}$ ). To test safety of trans-atrial pericardial access, 8 animals (4Fr Puncture group) underwent RAA puncture with a $4 \mathrm{Fr}(1.3 \mathrm{~mm}$ outer diameter) diagnostic multipurpose catheter and the back end of a $0.035^{\prime \prime}$ guidewire and 8 animals (2.8Fr Puncture group) underwent RAA puncture with a $2.8 \mathrm{Fr}(0.9 \mathrm{~mm}$ outer diameter) microcatheter (Excelsior, Boston Scientific) and the back end of a $0.018^{\prime \prime}$ guidewire (V18, Boston Scientific).

To test $\mathrm{CO}_{2}$ insufflation of the pericardial space to facilitate sub-xiphoid access, 7 animals (2.8Fr $+\mathrm{CO}_{2}$ group) underwent RAA puncture with a $2.8 \mathrm{Fr}$ microcatheter and the back end of a 0.018 " guidewire. The pericardium was then insufflated with $\mathrm{CO}_{2}$ and sub-xiphoid pericardial access was obtained. To test iodinated contrast-facilitated sub-xiphoid access, iopamidol (Isovue 300, Bracco) diluted to $25 \%$ in normal saline was infused through a $2.8 \mathrm{Fr}$ trans-atrial microcatheter using a power injector in 7 animals (2.8Fr + Contrast group). Figure 1 summarizes the experimental design.

In all animals, a 6Fr diagnostic multipurpose or angled-tip balloon wedge end-hole catheter was navigated to the RAA from the inferior vena cava using fluoroscopic guidance (Figure 2). Position at the tip of the RAA was confirmed with contrast angiography. The back end of a guidewire (either $0.035^{\prime \prime}$ or $0.018^{\prime \prime}$ ) was used to puncture through the wall of the RAA into the pericardial space. A catheter (4Fr multipurpose or $2.8 \mathrm{Fr}$ microcatheter) was then advanced over the wire into the pericardium. To exaggerate the risk of bleeding, unfractionated heparin (150-200IU/kg bodyweight) was administered to the first 16 animals to achieve a mean activated clotting time [16] of 383sec (range 263-517sec), before withdrawal of the trans-atrial catheter back into the right atrium.

In the $2.8 \mathrm{Fr}+\mathrm{CO}_{2}$ group, $100-150 \mathrm{cc}$ of $\mathrm{CO}_{2}$ (approximately $2-3 \mathrm{cc} / \mathrm{kg}$ bodyweight) was delivered into the pericardial space through the trans-atrial microcatheter to separate the visceral and parietal layers (target separation $10 \mathrm{~mm}$ ). Selective coronary angiography was performed before and after $\mathrm{CO}_{2}$ insufflation to monitor for coronary artery compression or spasm. Quantitative coronary angiography analysis was performed in the mid left anterior descending artery (LAD) and mid left circumflex artery (LCx) using Syngo QCA software (Siemens). Sub-xiphoid access was then obtained using a 20-gauge micro-puncture needle ( $0.8 \mathrm{~mm}$ outer diameter) and $0.018^{\prime \prime}$ guidewire using biplane fluoroscopic guidance. After sub-xiphoid access was obtained, the trans-atrial microcatheter was withdrawn back into the right atrium. 
In the $2.8 \mathrm{Fr}+$ Contrast group, $2-3 \mathrm{~mL} / \mathrm{kg}$ bodyweight of iodinated contrast diluted to $25 \%$ in normal saline was infused into the pericardial space through a trans-atrial microcatheter using a power injector at a rate of $1 \mathrm{ml} / \mathrm{sec}$ to minimize microcatheter tip whip. Sub-xiphoid access was obtained using a 20 -gauge micro-puncture needle and $0.018^{\prime \prime}$ guidewire.

All animals underwent serial MRI to monitor for pericardial effusion and/or tamponade after trans-atrial catheter withdrawal. We prefer MRI for this purpose because transthoracic echocardiography is suboptimal in swine. Contiguous true axial slices were acquired using a steady-state free precession (SSFP) sequence with breath hold and ECG gating. Slices were prescribed to include cardiac anatomy from base to apex to ensure coverage of the entirety of the pericardial space. Acquisition parameters were: repetition time (TR)/echo time (TE), 2.96/1.25msec; number of averages 2; acceleration factor (GRAPPA) 2; flip angle $80^{\circ}$; bandwidth $1157 \mathrm{~Hz} /$ pixel; field of view $300 \times 300 \mathrm{~mm}$; matrix $240 \times 128$ pixels; slice thickness $6 \mathrm{~mm}$; and slice distance $0 \mathrm{~mm}$. Pericardial and cardiac contours were drawn manually on each slice and the pericardial effusion volume was calculated by subtracting the total cardiac volume from the total pericardial volume using Syngo MR analysis software (Siemens).

Data were analysed using SPSS (v19.0, IBM) and reported as mean \pm standard deviation unless otherwise described. Mean differences were examined by $t$-test or one-way repeated measures ANOVA. A $p$ value $₫ .05$ was considered significant. To estimate the probability of an adverse event, the binomial confidence interval was calculated using a one-tailed adjusted Wald interval, and a point estimate using the LaPlace method. The 95\% confidence interval was calculated using an online tool (http://www.measuringusability.com/wald.htm). This same method was used to calculate the number of trials needed to achieve $95 \%$ confidence that the true event rate would be less than $1 \%$ and $0.1 \%$, based on the observed event rate.

\section{Results}

Pericardial access via RAA puncture was successfully obtained in all animals. Once the catheter position was confirmed in the RAA with angiography, exit into the pericardium took a few seconds only. The guidewire tracked along - but did not puncture - the parietal pericardium in all animals. In the 4Fr Puncture group (1.33mm puncture with 4Fr catheter) small pericardial effusions were observed in $5 / 8$ anticoagulated animals with mean volume $18.5 \mathrm{~mL}$ (range 7-76mL) $15 \mathrm{~min}$ after trans-atrial catheter withdrawal. Effusions did not enlarge over the following $2 \mathrm{hrs}$. There was an insignificant decline in systolic and diastolic blood pressures (Figure 3). There was no change in right atrial pressure. The largest effusion $(76 \mathrm{~mL})$ was observed in one animal with the highest ACT of $498 \mathrm{sec}$ before trans-atrial catheter withdrawal. Otherwise there was no correlation between ACT and effusion volume. In the 2.8Fr Puncture group, no effusions were observed despite full anticoagulation.

In the $2.8 \mathrm{Fr}+\mathrm{CO}_{2}$ group, pericardial $\mathrm{CO}_{2}$ insufflation effectively separated visceral and parietal layers anteriorly to the target separation of $10 \mathrm{~mm}$ in all 7 animals. This separation was highly visible on lateral plane fluoroscopy (Figure 4). A mild transient reduction in systolic blood pressure and increase in heart rate was observed during insufflation, but these did not reach statistical significance (Figure 3). Selective coronary angiography did not 
show any significant coronary artery compression or vasospasm up to $15 \mathrm{~min}$ post- $\mathrm{CO}_{2}$ insufflation (Figure 2). Minimum lumen diameters at baseline vs. with pericardial $\mathrm{CO}_{2}$ insufflation were 2.8 vs. $2.7 \mathrm{~mm}$ in the mid LAD and 2.7 vs. $2.5 \mathrm{~mm}$ in the mid LCx. Subxiphoid access with a micro-puncture needle aiming for the space created by the $\mathrm{CO}_{2}$ was technically easy, took under a minute and was successful in all 7 animals without complication (Figure 2 and Online Video Supplement).

The separation of pericardial layers enabled the needle to avoid contact with the myocardium altogether. Serial fluoroscopy after insufflation with $\mathrm{CO}_{2}$ showed pericardial $\mathrm{CO}_{2}$ spontaneously and completely resorbed within $15 \mathrm{mins}$ in all animals. No pericardial effusions were observed on MRI after withdrawal of the trans-atrial microcatheter.

Based on observing 0 effusions out of 15 trans-atrial access attempts with the $2.8 \mathrm{Fr}$ microcatheter (2.8Fr Puncture and $2.8 \mathrm{Fr}+\mathrm{CO}_{2}$ groups), the binomial confidence interval and best point estimate were calculated to be 0 to $18 \%$ (95\% CI) and 6\% respectively. In order to achieve $95 \%$ confidence that the true incidence of effusion is less than $1 \%$ and $0.1 \%$, we would need to perform 325 and 3150 observations each without any effusion, respectively.

In the $2.8 \mathrm{Fr}+$ Contrast group, infusion of dilute iodinated contrast effectively separated visceral and parietal pericardial layers posteriorly in all 7 animals. This separation was highly visible on lateral plane fluoroscopy (Figure 4), and created a target for easy and uncomplicated subxiphoid posterior pericardial access using a micro-puncture needle. However it did not separate the heart from the sternum for anterior pericardial access.

\section{Discussion}

\section{Sub-xiphoid 'dry' pericardial access}

The commonest complication to arise from sub-xiphoid 'dry' pericardial access is bleeding from right ventricle (RV) or coronary artery injury [5-7]. This can lead to tamponade and require surgical intervention. Unusual complications such as RV pseudoaneurysm or hepatic puncture with hematoma or abdominal bleeding have also been described [17]. Studies have not suggested an operator learning curve, as the complication rate does not appear to fall with time - even in expert hands. Strategies to improve the technique have been proposed and are elegantly summarized in a recent review article by Lim et al [10]. These strategies include suspending ventilation during pericardial puncture to prevent the heart from impaling itself on the needle, using micro-puncture or blunt scalloped-tip Tuohy epidural needles to reduce the risk of serious RV or coronary artery injury, integrating the needle with an electroanatomic mapping system for better tip navigation [18], or using live spectral analysis of the pressure waveform from the sub-xiphoid needle to detect the higherfrequency phasic signal characteristic of pericardial entry [19]. Importantly, none of these strategies specifically separate the heart from the potentially injurious needle.

The same studies also reported rates of failure to obtain access of 3-10\%, usually because of pericardial adhesions after prior open chest cardiac surgery or epicardial ablation. It is likely that the risk of RV injury is higher in these patients because the myocardium is tethered to 
the parietal pericardium. Adhesions do not necessarily preclude access because adhesion disruption with guidewires or catheters is possible [20].

Interestingly, the rate of RV puncture without bleeding in these studies was high (5-17\%) suggesting that puncturing the myocardium with a needle can be safe. Another single center study reported inadvertent RV puncture with an 18-gauge needle in fully anticoagulated patients but no bleeding was observed [4]. These observations support our hypothesis that puncturing the substantially lower pressure right atrium with a small microcatheter is safe and should also be safe in patients with elevated right atrial pressures. It is important to recognize that many of the serious bleeding complications caused by sub-xiphoid 'dry' pericardial access are due to lacerations caused by the heart beating against the sharp needle tip, rather than from a direct needle puncture.

\section{Trans-atrial 'dry' pericardial access}

In this paper, we describe a novel use of intentional trans-atrial pericardial access. Our study confirmed the finding of prior pre-clinical experiments: that accessing the pericardium by deliberately exiting through the RAA with small catheters is easy and safe [11, 12]. We found that a multipurpose diagnostic catheter enabled navigation to the tip of the RAA in most animals. In animals with prominent RAA trabeculation, an angled-tip balloon wedge end-hole catheter was preferred because the balloon prevented the catheter tip from becoming entangled in the trabeculae. Puncturing the RAA wall with the blunt back end of a guidewire (either $0.035^{\prime \prime}$ or $0.018^{\prime \prime}$ ) was simple. Advancing a small catheter into the pericardial space over the wire did not meet with resistance.

Even in the presence of full anticoagulation and the larger 4Fr trans-atrial catheter, we observed only small pericardial effusions with no evidence of tamponade physiology after catheter withdrawal. Spontaneous closure of small RAA punctures has been described previously, with small thrombi at the puncture site, focal inflammatory infiltrates and increased collagen deposition seen on histology [12]. We therefore tested whether using a micro- catheter could eliminate effusions altogether. With the smaller $2.8 \mathrm{Fr}$ microcatheter, we did not observe any effusions after trans-atrial microcatheter withdrawal in any of the 15 animals (2.8Fr Puncture and $2.8 \mathrm{Fr}+\mathrm{CO}_{2}$ groups).

We consider these findings, of zero effusions after 15 trans-atrial access attempts with $\mathrm{CO}_{2}$ insufflation, to be compelling. However, these data provide $95 \%$ confidence only that the true incidence of effusion is $18 \%$ or less. In order to achieve similar confidence that the true incidence of effusion is less than $1 \%$ would require 325 observations of 0 effusions, and if less than $0.1 \%$ would require 3150 such observations. Both are unattainable. Moreover we believe even a small pericardial effusion may be considered clinically acceptable in order to avoid more serious complications. Based on these observations, we have commenced enrollment in a clinical trial to test intentional right atrial exit to facilitate sub-xiphoid access to the anterior pericardial space in patients undergoing left atrial appendage suture ligation.

\section{Pericardial contrast media}

Pericardial iodinated contrast has been shown to facilitate navigation within the pericardial space [21]. Important structures such as atrial appendages, right ventricular outflow tract or 
atrioventricular groove can be delineated. However, contrast is heavier than water and so preferentially collects behind the heart when the patient is supine. This separates the layers of pericardium inferiorly and posteriorly and provides a target for the sub-xiphoid needle for access to the posterior pericardial space (Figure 4). However, because of its viscosity it is difficult to aspirate iodinated contrast - even diluted in normal saline - through the transatrial microcatheter. Contrast would need to be aspirated through a larger sheath after subxiphoid access is established.

$\mathrm{CO}_{2}$ is much lighter than water so collects anteriorly between the sternum and the heart when the patient is supine (Figure 4). It affords excellent visualization of the heart contour and provides a space to aim for with the sub-xiphoid needle. This space enables anterior pericardial access without contacting the anterior surface of the heart. Anterior access is a prerequisite for certain interventions such as percutaneous left atrial appendage suture ligation. The space created by the $\mathrm{CO}_{2}$ reduces the risk of lacerating the $\mathrm{RV}$ free wall or a coronary artery, usually caused by the heart beating against the sharp needle tip. An additional advantage of $\mathrm{CO}_{2}$ is that if, for any reason, sub-xiphoid access cannot be obtained, then the $\mathrm{CO}_{2}$ introduced does not need to be aspirated, but instead will resorb spontaneously.

Insufflating body cavities with $\mathrm{CO}_{2}$ is safe. Because $\mathrm{CO}_{2}$ solubility in blood is high, the reported incidence of significant $\mathrm{CO}_{2}$ gas embolism from pneumoperitoneum during laparoscopy is less than $0.002 \%$ [22]. Flooding the surgical field with $\mathrm{CO}_{2}$ while coming off cardiopulmonary bypass has been shown to reduce the incidence of air embolism [23]. Some studies of laparoscopic abdominal surgery have documented hypercarbia, thought to be due to $\mathrm{CO}_{2}$ absorption from the peritoneal cavity and tissues [24]. However much of this effect is likely attributable to hypoventilation caused by diaphragmatic splinting, which is unlikely to occur with pericardial $\mathrm{CO}_{2}$ insufflation. In this study, pericardial $\mathrm{CO}_{2}$ insufflation was very well tolerated. A transient reduction in systolic blood pressure was seen (mean decrease from baseline $6 \mathrm{mmHg}$ or $7.7 \%$ ) but this was reversible with spontaneous absorption of the pericardial $\mathrm{CO}_{2}$. We did not observe any evidence of cardiac tamponade in any animal. Fluid or gas in the pericardial space can interfere with epicardial interventions, for example by elevating defibrillation thresholds during electrophysiological interventions [25].

However, this concern is not relevant to the present technique because pericardial iodinated contrast or $\mathrm{CO}_{2}$ can be easily aspirated through the sub-xiphoid access port and moreover, $\mathrm{CO}_{2}$ will resorb rapidly and spontaneously.

\section{Conclusion}

Intentional right atrial exit to access the 'dry' pericardium with small catheters was technically easy and safe in this pre-clinical study. Iodinated contrast or $\mathrm{CO}_{2}$ infused through a trans-atrial catheter created a target that enhanced the safety of sub-xiphoid access so large sheaths could be introduced for electrophysiological or structural heart interventions. Iodinated contrast collected posteriorly, facilitating sub-xiphoid access to the posterior pericardium, whereas $\mathrm{CO}_{2}$ separated the $\mathrm{RV}$ free wall from the sternum facilitating sub-xiphoid access to the anterior pericardium. This separation should reduce the risk of RV or coronary artery laceration. Trans-atrial microcatheters, which are smaller than 18-gauge 
needles, were withdrawn uneventfully despite full anticoagulation. Based on these preclinical findings, we commenced enrollment in a clinical trial to test this new technique in patients undergoing left atrial appendage suture ligation.

\title{
Supplementary Material
}

Refer to Web version on PubMed Central for supplementary material.

\section{Acknowledgments}

\author{
We thank Katherine Lucas and Joni Taylor for their help with animal experiments. \\ Funding information \\ This work was supported by the Division of Intramural Research, National Heart Lung and Blood Institute, \\ National Institutes of Health (Z01-HL006040-01 and Z01-HL005062).
}

\section{References}

1. Bartus K, Bednarek J, Myc J, Kapelak B, Sadowski J, Lelakowski J, Yakubov SJ, Lee RJ. Feasibility of closed-chest ligation of the left atrial appendage in humans. Heart Rhythm. 2011; 8(2):188-93. [PubMed: 21050893]

2. Sosa E, Scanavacca M, d'Avila A, Pilleggi F. A new technique to perform epicardial mapping in the electrophysiology laboratory. J Cardiovasc Electrophysiol. 1996; 7(6):531-6. [PubMed: 8743758]

3. Sosa E, Scanavacca M, D’Avila A, Piccioni J, Sanchez O, Velarde JL, Silva M, Reolao B. Endocardial and epicardial ablation guided by nonsurgical transthoracic epicardial mapping to treat recurrent ventricular tachycardia. J Cardiovasc Electrophysiol. 1998; 9(3):229-39. [PubMed: 9580377]

4. Page SP, Duncan ER, Thomas G, Ginks MR, Earley MJ, Sporton SC, Dhinoja M, Schilling RJ. Epicardial catheter ablation for ventricular tachycardia in heparinized patients. Europace. 2013; 15(2):284-9. [PubMed: 23002196]

5. Della Bella P, Brugada J, Zeppenfeld K, Merino J, Neuzil P, Maury P, Maccabelli G, Vergara P, Baratto F, Berruezo A, Wijnmaalen AP. Epicardial ablation for ventricular tachycardia: a European multicenter study. Circ Arrhythm Electrophysiol. 2011; 4(5):653-9. [PubMed: 21841191]

6. Sacher F, Roberts-Thomson K, Maury P, Tedrow U, Nault I, Steven D, Hocini M, Koplan B, Leroux L, Derval N, Seiler J, Wright MJ, Epstein L, Haissaguerre M, Jais P, Stevenson WG. Epicardial ventricular tachycardia ablation a multicenter safety study. J Am Coll Cardiol. 2010; 55(21):2366-72. [PubMed: 20488308]

7. Tung R, Michowitz Y, Yu R, Mathuria N, Vaseghi M, Buch E, Bradfield J, Fujimura O, Gima J, Discepolo W, Mandapati R, Shivkumar K. Epicardial ablation of ventricular tachycardia: an institutional experience of safety and efficacy. Heart Rhythm. 2013; 10(4):490-8. [PubMed: 23246598]

8. Price MJ, Gibson DN, Yakubov SJ, Schultz JC, Di Biase L, Natale A, Burkhardt JD, Pershad A, Byrne TJ, Gidney B, Aragon JR, Goldstein J, Moulton K, Patel T, Knight B, Lin AC, Valderrábano M. Early Safety and Efficacy of Percutaneous Left Atrial Appendage Suture Ligation: Results From the U.S. Transcatheter LAA Ligation Consortium. J Am Coll Cardiol. 2014; 64(6):565-572. [PubMed: 25104525]

9. Miller MA, Gangireddy SR, Doshi SK, Aryana A, Koruth JS, Sennhauser S, d'Avila A, Dukkipati SR, Neuzil P, Reddy VY. Multicenter study on acute and long-term safety and efficacy of percutaneous left atrial appendage closure using an epicardial suture snaring device. Heart Rhythm. 2014

10. Lim HS, Sacher F, Cochet H, Berte B, Yamashita S, Mahida S, Zellerhoff S, Komatsu Y, Denis A, Derval N, Hocini M, Haissaguerre M, Jais P. Safety and prevention of complications during 
percutaneous epicardial access for the ablation of cardiac arrhythmias. Heart Rhythm. 2014; 11(9): 1658-65. [PubMed: 24909982]

11. Verrier RL, Waxman S, Lovett EG, Moreno R. Transatrial access to the normal pericardial space: a novel approach for diagnostic sampling, pericardiocentesis, and therapeutic interventions. Circulation. 1998; 98(21):2331-3. [PubMed: 9826322]

12. Waxman S, Pulerwitz TC, Rowe KA, Quist WC, Verrier RL. Preclinical safety testing of percutaneous transatrial access to the normal pericardial space for local cardiac drug delivery and diagnostic sampling. Catheter Cardiovasc Interv. 2000; 49(4):472-7. [PubMed: 10751782]

13. Mickelsen SR, Ashikaga H, DeSilva R, Raval AN, McVeigh E, Kusumoto F. Transvenous access to the pericardial space: an approach to epicardial lead implantation for cardiac resynchronization therapy. Pacing Clin Electrophysiol. 2005; 28(10):1018-24. [PubMed: 16221257]

14. Scanavacca MI, Venancio AC, Pisani CF, Lara S, Hachul D, Darrieux F, Hardy C, Paola E, Aiello VD, Mahapatra S, Sosa E. Percutaneous transatrial access to the pericardial space for epicardial mapping and ablation. Circ Arrhythm Electrophysiol. 2011; 4(3):331-6. [PubMed: 21430128]

15. Rogers T, Ratnayaka K, Sonmez M, Franson DN, Schenke WH, Mazal JR, Kocaturk O, Chen MY, Faranesh AZ, Lederman RJ. Trans-Auricular Intra-Pericardial Tricuspid Annuloplasty. 2014 Under Review.

16. The Task Force on the management of stable coronary artery disease of the European Society of Cardiology. 2013 ESC guidelines on the management of stable coronary artery disease. Eur Heart J. 2013

17. Koruth JS, Aryana A, Dukkipati SR, Pak HN, Kim YH, Sosa EA, Scanavacca M, Mahapatra S, Ailawadi G, Reddy VY, d'Avila A. Unusual complications of percutaneous epicardial access and epicardial mapping and ablation of cardiac arrhythmias. Circ Arrhythm Electrophysiol. 2011; 4(6): 882-8. [PubMed: 22007036]

18. Bradfield JS, Tung R, Boyle NG, Buch E, Shivkumar K. Our approach to minimize risk of epicardial access: standard techniques with the addition of electroanatomic mapping guidance. $\mathbf{J}$ Cardiovasc Electrophysiol. 2013; 24(6):723-7. [PubMed: 23279311]

19. Mahapatra S, Tucker-Schwartz J, Wiggins D, Gillies GT, Mason PK, McDaniel G, Lapar DJ, Stemland C, Sosa E, Ferguson JD, Bunch TJ, Ailawadi G, Scanavacca M. Pressure frequency characteristics of the pericardial space and thorax during subxiphoid access for epicardial ventricular tachycardia ablation. Heart Rhythm. 2010; 7(5):604-9. [PubMed: 20156612]

20. Tschabrunn CM, Haqqani HM, Zado ES, Marchlinski FE. Repeat percutaneous epicardial mapping and ablation of ventricular tachycardia: safety and outcome. J Cardiovasc Electrophysiol. 2012; 23(7):744-9. [PubMed: 22353308]

21. Cohn WE, Winkler JA, Tuzun E, Hjelle A, Bassett K, Ahmad A, Frazier OH. Contrast pericardiography facilitates intrapericardial navigation under fluoroscopy. Ann Thorac Surg. 2010; 90(5):1537-40. [PubMed: 20971258]

22. Cueto-Garcia, J.; Jacobs, M.; Gagner, M. Laparoscopic Surgery. McGraw-Hill; 2003.

23. Ng WS, Rosen M. Carbon dioxide in the prevention of air embolism during open-heart surgery. Thorax. 1968; 23(2):194-6. [PubMed: 5654077]

24. Hirvonen EA, Nuutinen LS, Kauko M. Ventilatory effects, blood gas changes, and oxygen consumption during laparoscopic hysterectomy. Anesth Analg. 1995; 80(5):961-6. [PubMed: 7726439]

25. Yamada T, McElderry HT, Platonov M, Doppalapudi H, Kay GN. Aspirated air in the pericardial space during epicardial catheterization may elevate the defibrillation threshold. Int J Cardiol. 2009; 135(1):e34-5. [PubMed: 18593642] 


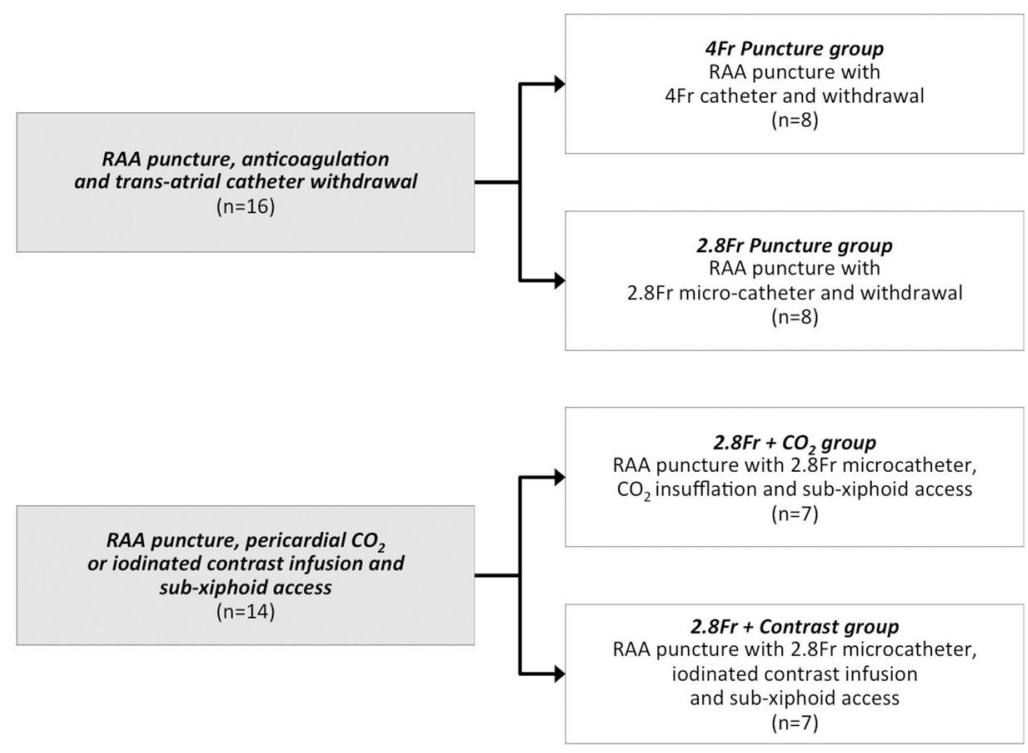

Figure 1. Experimental design

4Fr Puncture group: RAA puncture with 4Fr $(1.3 \mathrm{~mm})$ catheter then withdrawal after heparinization; 2.8Fr Puncture group: RAA puncture with $2.8 \mathrm{Fr}(0.9 \mathrm{~mm})$ microcatheter then withdrawal after heparinization; $2.8 \mathrm{Fr}+\mathrm{CO}_{2}$ group: RAA puncture with $2.8 \mathrm{Fr}$ microcatheter, insufflation with $\mathrm{CO}_{2}$, sub-xiphoid access and RAA microcatheter withdrawal after heparinization. $2.8 \mathrm{Fr}+$ Contrast group: RAA puncture with $2.8 \mathrm{Fr}$ microcatheter and infusion of iodinated contrast. RAA: right atrial appendage. 

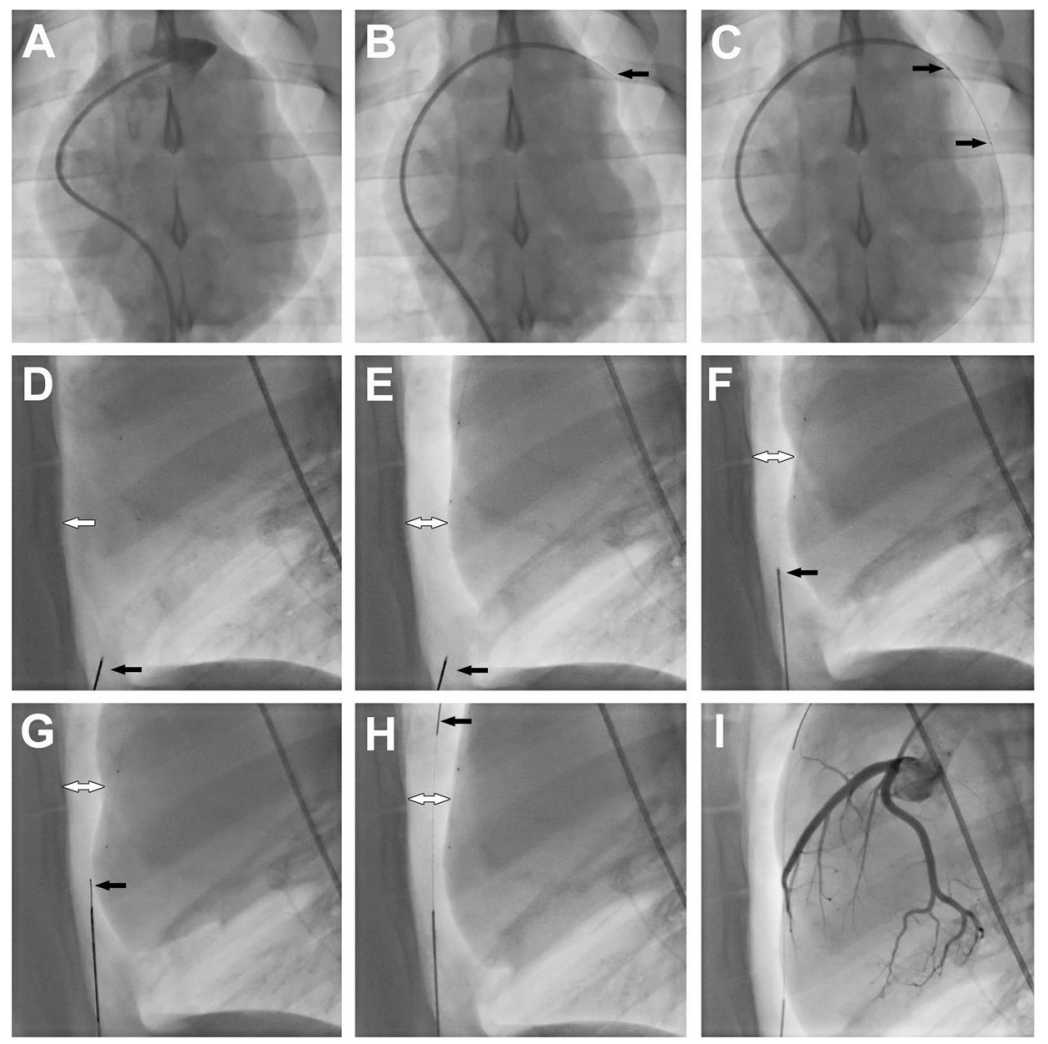

Figure 2. Procedural steps

(A) Right atrial appendage angiogram. (B) Trans-atrial puncture with the back end of a $0.018^{\prime \prime}$ guidewire. (C) $2.8 \mathrm{Fr}$ microcatheter advanced over the $0.018^{\prime \prime}$ guidewire into the pericardial space. Black arrows show radiopaque markers on the microcatheter. (D) Lateral plane fluoroscopy showing close apposition of anterior surface of the myocardium and the sternum (white arrow). Black arrow shows the sub-xiphoid needle. (E) Pericardial insufflation with $\mathrm{CO}_{2}$. Black arrow shows the sub-xiphoid needle. White arrow shows separation of pericardial layers. (F) Sub-xiphoid needle positioned to enter the pericardial space. Black arrow marks the tenting of the pericardium. (G) $0.018^{\prime \prime}$ guidewire (black arrow) introduced through the sub-xiphoid needle into the pericardium. $(\mathrm{H}) 0.018^{\prime \prime}$ guidewire (black arrow) tracked along anterior pericardial surface to the apex of the heart. (I) Coronary angiogram to assess for coronary artery compression or spasm. 


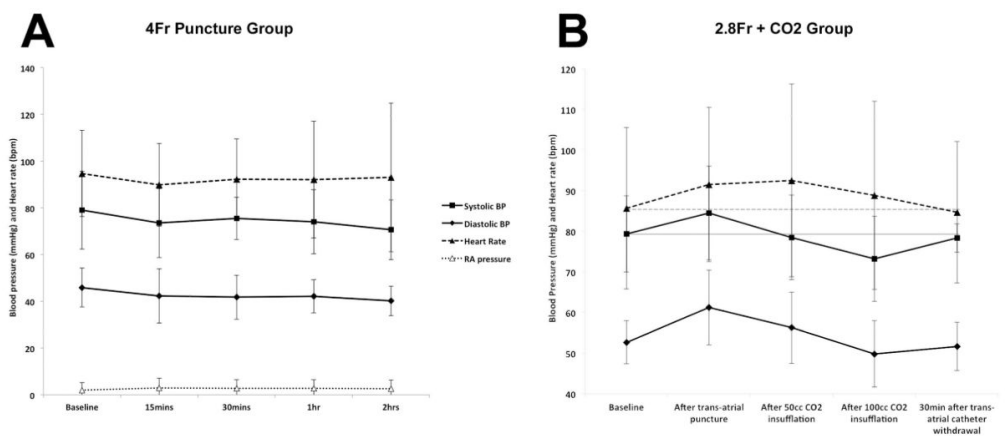

Figure 3. Hemodynamic parameters

(A) Hemodynamic parameters do not change significantly before trans-atrial pericardial access with 4Fr catheter, and 15, 30, 60 and 120min after trans-atrial catheter withdrawal. (B) Hemodynamic parameters before trans-atrial pericardial access with $2.8 \mathrm{Fr}$ microcatheter, after pericardial $\mathrm{CO}_{2}$ insufflation, and 30min after sub-xiphoid access and trans-atrial catheter withdrawal. Grey horizontal solid and dashed lines indicate baseline systolic blood pressure and heart rate respectively. BP: blood pressure. 

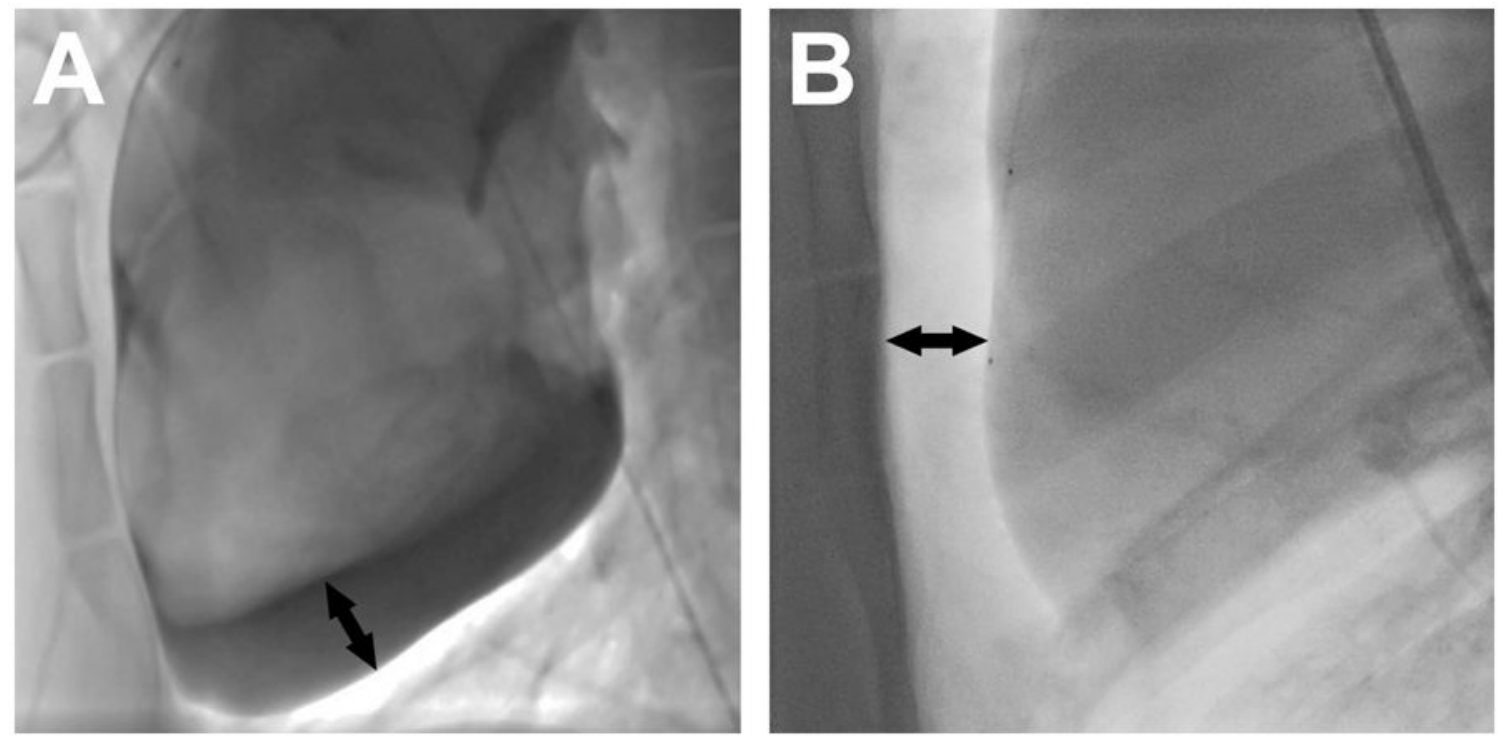

Figure 4. Pericardial iodinated contrast vs. carbon dioxide

Lateral plane fluoroscopy in a supine animal of (A) iodinated contrast and (B) $\mathrm{CO}_{2}$ in the pericardium, both introduced through a trans-atrial microcatheter. Black arrows show the separation of the inferior surface of the heart from the pericardium in (A) and of the anterior surface of the heart from the sternum in (B). 